\title{
THE CHROMATIC CONVERGENCE THEOREM AND A TOWER IN ALGEBRAIC $K$-THEORY
}

\author{
J. E. MCCLURE AND R. E. STAFFELDT \\ (Communicated by Frederick R. Cohen)
}

\begin{abstract}
In this note we show how the chromatic convergence theorem of Hopkins and Ravenel implies that a tower of relative algebraic $K$-theories constructed by Waldhausen converges to the $p$-local part of the algebraic $K$-theory of the one-point space relative to the $K$-theory of the integers. The notion of convergence used here is made precise using the language of pro-homotopy theory.
\end{abstract}

\section{BACKGROUND}

In 1982 Waldhausen [8] described a method for investigating the fiber of the map

$$
A(*) \rightarrow K(\mathbf{Z})
$$

here $A(*)$ is the $A$-theory of the one-point space, which can also be thought of as the algebraic $K$-theory of the ring space $Q S^{0}$ or as the algebraic $K$-theory of the category of spectra of finite type. He began by showing [8, p. 186] that the $p$-local part of this fiber is the fiber of a map

$$
A(*, p) \rightarrow K\left(\mathbf{Z}_{(p)}\right),
$$

where $A(*, p)$ is the $K$-theory of the category of $p$-local spectra of finite type. He then used the chromatic tower in stable homotopy to construct a tower of algebraic $K$-theory spaces

$$
\widetilde{A}(*, p, n) \rightarrow \cdots \rightarrow \tilde{A}(*, p, 1) \rightarrow \tilde{A}(*, p, 0)=K\left(\mathbf{Z}_{(p)}\right),
$$

which he called the integral localization tower, and a map from $A(*, p)$ to this tower. The idea was to consider the integral localization tower as an approximation to $A(*, p)$, but Waldhausen did not show that it actually converged to $A(*, p)$ in any sense. The purpose of the present note is to use the chromatic

Received by the editors November 25, 1991.

1991 Mathematics Subject Classification. Primary 55P60, 19D10; Secondary 55Q52, 55S35.

Key words and phrases. Algebraic $K$-theory of spaces, chromatic filtration of stable homotopy, weak pro-homotopy equivalence.

The first author was partially supported by NSF grant DMS- 8803279 and by the Sonderforschungsbereich 343 , Bielefeld. The second author was partially supported by the Sonderforschungsbereich 343, Bielefeld. 
convergence theorem of Hopkins and Ravenel [3] to show that this tower does in fact converge to $A(*, p)$; specifically, we show that the map

$$
\{A(*, p)\} \rightarrow\{\tilde{A}(*, p, n)\}
$$

from the constant tower to the integral localization tower is a weak pro-homotopy equivalence, a definition recalled in the next section. It follows that the map of towers

$$
\left\{\operatorname{fibre}\left(A(*, p) \rightarrow K\left(\mathbf{Z}_{(p)}\right)\right)\right\} \rightarrow\left\{\operatorname{fibre}\left(\tilde{A}(*, p, n) \rightarrow K\left(\mathbf{Z}_{(p)}\right)\right)\right\}
$$

is also a weak pro-homotopy equivalence.

In the interest of readability, we review in this section the definitions of the chromatic tower and of Waldhausen's tower before proceeding to the proof in the next section.

First recall the notion of Bousfield localization of spectra. Properties of Bousfield localization are also catalogued in [6, 8].

Definition 1.1. Let $E_{*}$ be a generalized homology theory. A spectrum $Y$ is $E_{*}$-local if whenever a map $f: X_{1} \rightarrow X_{2}$ is such that $E_{*}(f)$ is an isomorphism, the induced map $\left[X_{1}, Y\right] \leftarrow\left[X_{2}, Y\right]$ is also an isomorphism.

An $E_{*}$-localization of a spectrum $X$ is a map $\eta$ from $X$ to an $E_{*}$-local spectrum $X_{E}$ such that $E_{*}(\eta)$ is an isomorphism.

Not only do $E_{*}$-localizations exist, but they also have extremely good functorial properties, recorded in the following omnibus theorem due to Bousfield [1].

Theorem 1.2. For any homology theory $E_{*}$ and any spectrum $X$, the $E_{*}$ localization of $X$ exists and is functorial in $X$.

Emphasizing this functoriality, the notation $X_{E}=L_{E} X$ is standard and will be used in this paper.

Underlying the chromatic tower is the $p$-local homology theory $B P_{*}$ whose coefficient ring is

$$
B P_{*}=\mathbf{Z}_{(p)}\left[v_{1}, v_{2}, \ldots, v_{n}, \ldots\right],
$$

where the degree of $v_{n}$ is $2\left(p^{n}-1\right)$, and the actual starting point for the construction is the family of theories $E(n)_{*}, n \geq 1$, for which the coefficient rings are

$$
E(n)_{*}=\mathbf{Z}_{(p)}\left[v_{1}, v_{2}, \ldots, v_{n}, v_{n}^{-1}\right] .
$$

Original versions of the following definitions are found in [6], beginning with $\S 5$.

Definition 1.3. For a $p$-local spectrum $X$ and $n \geq 1$, put $L_{n} X=L_{E(n)} X$, the $E(n)_{*}$-localization of $X$. For $n=0$ put $L_{0} X$ equal to the rationalization of $X$.

According to [6] there are natural transformations of localization functors $L_{n} \rightarrow L_{n-1}$ compatible with the structure maps $X \rightarrow L_{n} X$, and we may make the following definition.

Definition 1.4. The chromatic tower for a $p$-local spectrum $X$ is the inverse system

$$
L_{0} X \leftarrow L_{1} X \leftarrow \cdots \leftarrow L_{n} X \leftarrow \cdots .
$$


The structure maps $X \rightarrow L_{n} X$ combine to give a natural map of towers of spectra $\{X\} \rightarrow\left\{L_{n} X\right\}$ from the constant tower on $X$ to the chromatic tower. Finally, the chromatic filtration of $\pi_{*} X$ is given by $\operatorname{ker}\left(\pi_{*} X \rightarrow \pi_{*} L_{n} X\right)$.

In the rest of this section we outline Waldhausen's construction of the integral localization tower, as given in [8, pp. 188-189]. One arrives at the starting point for the construction as follows. By specializing the chromatic tower to the case $X=S$, the sphere spectrum localized at $p$, by replacing the Eilenberg-Mac Lane spectrum $K \mathbf{Q}$ at the 0th level of the tower with the Eilenberg-Mac Lane spectrum $K \mathbf{Z}_{(p)}$, and by then applying the functor $\Omega^{\infty}$, which assigns to a spectrum its associated infinite loopspace, one obtains from the map of towers of spectra a map of towers of spaces $\{Q S\} \rightarrow\left\{Q S_{(n)}\right\}$, where we have adopted Waldhausen's notation for the spaces involved: $\Omega^{\infty} S=Q S, \Omega^{\infty} L_{n} S=Q S_{(n)}$, and $\mathbf{Z}_{(p)}=Q S_{(0)}$. As explained and hypothesized in [8], essential properties of the right-hand tower and of the map between the towers follow from the hypothesis that the localization functor $L_{n}$ has the property of being a smashing localization functor, a property of $L_{n}$ proven in [3]. These properties are that the spaces $Q S_{(n)}$ are ring spaces and that the maps in the tower are maps of ring spaces, as well as the maps $Q S \rightarrow Q S_{(n)}$, for each $n$.

With this information in hand, the construction of the tower of $K$-theories proceeds through the following steps. First let $M_{k}\left(Q S_{(n)}\right)$ denote the multiplicative $H$-space of $k \times k$ matrices over $Q S_{(n)}$. For $n \geq 1$, the monoid of connected components is $M_{k}\left(\pi_{0} Q S_{(n)}\right)$, and we define $\widehat{G L}_{k}\left(Q S_{(n)}\right)$ by pulling back over the inclusion of the invertible $k \times k$ matrices into all $k \times k$ matrices:

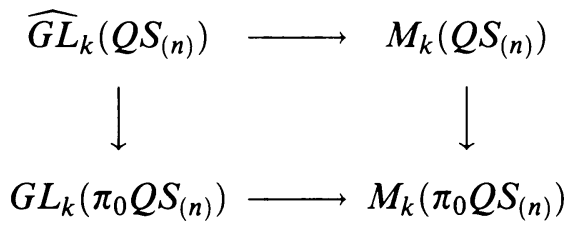

Waldhausen then shows that the $H$-space $\widehat{G L}_{k}\left(Q S_{(n)}\right)$ has a classifying space $B \widehat{G L}_{k}\left(Q S_{(n)}\right)$ and defines $B \widehat{G L}\left(Q S_{(n)}\right)$ by taking the colimit of these spaces as $k$ tends to infinity. He then obtains for each $n$ spaces and maps compatible with varying $n$,

$$
B \widehat{G L}(Q S) \rightarrow B \widehat{G L}\left(Q S_{(n)}\right)
$$

on which he performs the plus construction. All of this is recorded in the following diagram of maps of towers:

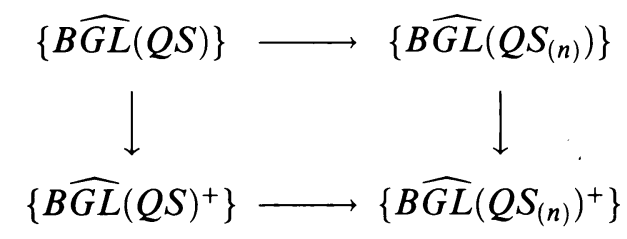

Finally he defines

$$
\widetilde{A}(*, p, n)=\mathbf{Z} \times B \widehat{B L}\left(Q S_{(n)}\right)^{+},
$$

where the factor $\mathbf{Z}$ represents the class group of the free modules over the ring $\pi_{0} Q S_{(n)}$, and similarly

$$
A(*, p)=\mathbf{Z} \times B \widehat{B L}(Q S)^{+}
$$


In the next section we consider the map of towers

$$
\{A(*, p)\} \rightarrow\{\tilde{A}(*, p, n)\},
$$

which is of course just the bottom row of the above diagram crossed with a copy of the integers.

Note added in proof. Waldhausen has pointed out to us that the localization functor $L_{n}$ used in our paper does not quite agree with the functor called $L_{n}$ in [8], and so the connection between our paper and [8] is less close than we have claimed in the introduction. The point is that there are two versions of $L_{n}$, namely the Bousfield localization (which we have used) and the functor which Ravenel [9] denotes by $L_{n}^{f}$. All of the results of [8] that we have actually used are valid for both variants, but for [8] as a whole one must use $L_{n}^{f}$. Thus the results in our paper are true as stated but probably not interesting from the point of view of algebraic $K$-theory. On the other hand, the full connection between our paper and [8] would be restored if it were known that $L_{n}^{f}$ satisfies a chromatic convergence property-this is at present an open question.

\section{THE MAIN RESULT}

In this section we recall what we need from pro-homotopy theory, state the chromatic convergence theorem, and prove our main result. First we recall from $[2$, p. 73$]$ the definition underlying the notion of pro-homotopy equivalence.

Definition 2.1. A map $f:\left\{G_{n}\right\} \rightarrow\left\{H_{n}\right\}$ between two towers (i.e., inverse systems) of groups is called a pro-isomorphism if, for every group $B, f$ induces an isomorphism

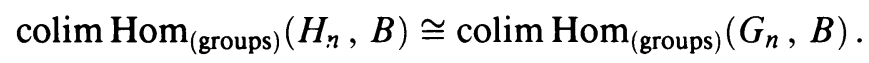

It is easily seen that the condition that $f$ induces an isomorphism of inverse limits is necessary but not sufficient for $f$ to be a pro-isomorphism. It is also important to observe that the definition is equivalent to the condition that, for every $n$, there is an $n^{\prime} \geq n$ and a homomorphism $H_{n^{\prime}} \rightarrow G_{n}$ such that the diagram

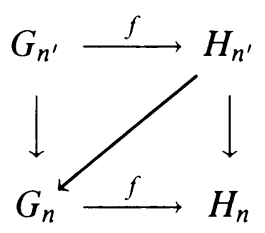

commutes.

Next we let $f:\left\{X_{n}\right\} \rightarrow\left\{Y_{n}\right\}$ be a map of towers of pointed connected spaces. From [2, p. 76] we take the following notion.

Definition 2.2. The map of towers of connected pointed spaces $f:\left\{X_{n}\right\} \rightarrow\left\{Y_{n}\right\}$ is a weak pro-homotopy equivalence if the induced maps $\left\{\pi_{q} X_{n}\right\} \rightarrow\left\{\pi_{q} Y_{n}\right\}$ are pro-isomorphisms for each $q \geq 1$.

We record the following useful recognition criterion for weak pro-homotopy equivalences, which should be thought of as a Whitehead theorem [7, p. 399] in the pro-category. This lemma seems to be well known, but we could not find it explicitly stated in the literature. ${ }^{1}$

${ }^{1}$ The authors would like to thank the referee for simplifying our original argument. 
Lemma 2.3. Let $f:\left\{X_{n}\right\} \rightarrow\left\{Y_{n}\right\}$ be a map of towers of connected pointed spaces such that $f_{*}:\left\{\pi_{1} X_{n}\right\} \rightarrow\left\{\pi_{1} Y_{n}\right\}$ is a pro-isomorphism and

$$
f^{*}: \operatorname{colim} H^{*}\left(Y_{n} ; N\right) \stackrel{\cong}{\rightrightarrows} \operatorname{colim} H^{*}\left(X_{n} ; N\right)
$$

for any $\pi_{1} Y_{k}$-module $N$ where $k \geq 0$. Then $f$ is a weak pro-homotopy equivalence.

Proof. This is actually an easy consequence of Lemma 4.3 of [2, p. 80], which says that, under the stated conditions and for any fibrant space $V$ and integer $s \geq 0$, the map between direct systems of sets of homotopy classes of maps induced by $f$ passes to a bijection

$$
\operatorname{colim}\left[X_{n}, V^{(s)}\right] \leftarrow \operatorname{colim}\left[Y_{n}, V^{(s)}\right],
$$

where $V^{(s)}$ is the $s$ th Postnikov section of $V$ as in [5, p. 32]. Whenever $A$ is a pointed connected space and $n \geq s$, obstruction theory, or even consideration of the Postnikov section construction, shows that the canonical map $A \rightarrow A^{(n)}$ induces a bijection

$$
\left[A^{(n)}, V^{(s)}\right] \rightarrow\left[A, V^{(s)}\right],
$$

so $f$ also induces a bijection

$$
\operatorname{colim}\left[X_{n}^{(n)}, V^{(s)}\right] \leftarrow \operatorname{colim}\left[Y_{n}^{(n)}, V^{(s)}\right] .
$$

Using this fact and specializing $V^{(s)}$ in various ways, one sees that for any $n$ there is $n^{\prime}>n$ and a map $Y_{n^{\prime}}^{\left(n^{\prime}\right)} \rightarrow X_{n}^{(n)}$ such that in the diagram

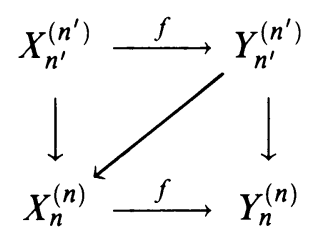

both triangles commute up to homotopy. In other words, $\left\{X_{n}^{(n)}\right\} \rightarrow\left\{Y_{n}^{(n)}\right\}$ is a pro-isomorphism of towers over the pointed homotopy category. (Compare Definition 2.1.) Now Proposition 3.3 of [2, p. 77] allows us to conclude that $\left\{X_{n}\right\} \rightarrow\left\{Y_{n}\right\}$ is a weak pro-homotopy equivalence, as required.

In [1] one finds the following extension of Definition 2.2 to spectra.

Definition 2.4. The map of towers of spectra $f:\left\{X_{n}\right\} \rightarrow\left\{Y_{n}\right\}$ is a weak prohomotopy equivalence if the map between towers $\left\{\pi_{q} X_{n}\right\} \rightarrow\left\{\pi_{q} Y_{n}\right\}$ is a proisomorphism of groups for each integer $q$.

With this terminology we may now state the chromatic convergence theorem proved in [3].

Theorem 2.5. For the suspension spectrum $\Sigma^{\infty} X$ of a p-local finite $C W$-complex $X$, the chromatic tower of Definition 1.4 converges in the sense that the map

$$
\left\{\Sigma^{\infty} X\right\} \rightarrow\left\{L_{n} \Sigma^{\infty} X\right\}
$$

from the constant tower to the chromatic tower is a weak pro-homotopy equivalence of spectra.

We will also need the following simple fact about pro-isomorphisms. 
Lemma 2.6. Let $f:\left\{R_{n}\right\} \rightarrow\left\{R_{n}^{\prime}\right\}$ be a map of towers of rings in which each map

$$
f: R_{n} \rightarrow R_{n}^{\prime}
$$

is an injective ring homomorphism. Then $f$ is a pro-isomorphism of rings if it is a pro-isomorphism of groups.

Proof. Since $f$ is a pro-isomorphism of groups, we have for each $n$ an integer $n^{\prime} \geq n$ and a group homomorphism $R_{n^{\prime}}^{\prime} \rightarrow R_{n}$ such that the diagram

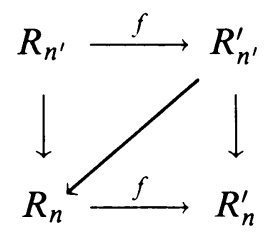

commutes. The thing to verify is that the diagonal arrow is actually a ring homomorphism, but this is a simple consequence of the injectivity of the lower arrow and the fact that the horizontal and vertical arrows are assumed to preserve products.

The following corollary is the starting point for the proof of our main theorem. It is proved by applying Theorem 2.5 when $X$ is a $p$-local sphere, passing to associated infinite loopspaces, and using the preceding lemma.

Corollary 2.7. Consider the map of towers of infinite loop spaces

$$
\{Q S\} \rightarrow\left\{Q S_{(n)}\right\}
$$

from the constant tower on $Q S$ to the chromatic tower.

(a) At the level of $\pi_{0}$ the induced map of towers of rings

$$
\mathbf{Z}_{(p)} \rightarrow\left\{\pi_{0} Q S_{(n)}\right\}
$$

is a pro-isomorphism.

(b) Restricted to the 0-components, the map of towers is a weak pro-homotopy equivalence of spaces.

Proof. For part (a) we need to observe that the map $\mathbf{Z}_{(p)} \rightarrow \pi_{0} Q S_{(n)}$ is always a monomorphism because the rationalization map $\mathbf{Z}_{(p)} \rightarrow \pi_{0} Q S_{(0)}$ factors through it. Part (a) now follows from Theorem 2.5 and Lemma 2.6, and part (b) is immediate from 2.5.

Remark. In this section we have somewhat awkward statements because the notion of weak pro-homotopy equivalence has only been defined here for connected spaces and the spaces involved here are by definition disconnected; however, all spaces with which we are dealing are $H$-spaces with an abelian group of components, so any two components of one of these spaces are actually homotopy equivalent.

Now we can prove our main result.

Theorem 2.8. The map of towers of spaces

$$
\{A(*, p)\} \rightarrow\{\tilde{A}(*, p, n)\}
$$


from the constant tower on the algebraic $K$-theory of the $p$-local finite spectra to the integral localization tower is a weak pro-homotopy equivalence on connected components.

Proof. We return to the diagram of the previous section:

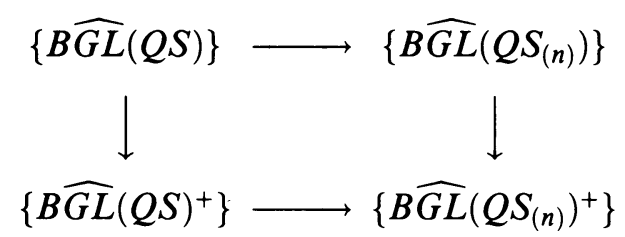

For brevity write $X=B \widehat{G L}(Q S), Y_{n}=B \widehat{G L}\left(Q S_{(n)}\right), X^{+}=B \widehat{G L}(Q S)^{+}$, and $Y_{n}^{+}=B \widehat{G L}\left(Q S_{(n)}\right)^{+}$so that the diagram becomes:

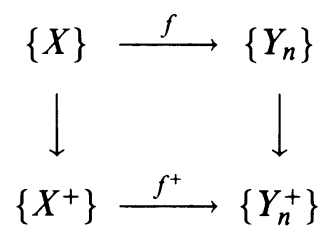

To prove the theorem we have to show that $f^{+}:\left\{X^{+}\right\} \rightarrow\left\{Y_{n}^{+}\right\}$is a weak pro-homotopy equivalence.

To start, notice that $f:\{X\} \rightarrow\left\{Y_{n}\right\}$ is a weak pro-homotopy equivalence. The induced map on fundamental groups is

$$
\left\{G L\left(\mathbf{Z}_{(p)}\right)\right\} \rightarrow\left\{G L\left(\pi_{0} Q S_{(n)}\right)\right\} .
$$

Now part (a) of Corollary 2.7 says we have a pro-isomorphism of rings

$$
\mathbf{Z}_{(p)} \rightarrow\left\{\pi_{0} Q S_{(n)}\right\}
$$

and the functor $G L$ turns a pro-isomorphism of rings into a pro-isomorphism of groups (use the characterization of pro-isomorphism given at the beginning of the section to see this). Thus we have proved that $f$ induces a pro-isomorphism at the level of $\pi_{1}$. At the level of $\pi_{q}$ for $q \geq 2$, recall that $\pi_{q} X=M\left(\pi_{q-1} Q S\right)$ and $\pi_{q} Y_{n}=M\left(\pi_{q-1} Q S_{(n)}\right)$, where for an abelian group $A, M(A)$ is the abelian group of infinite matrices over $A$, almost all of whose entries are 0 , and apply part (b) of Corollary 2.7 to see that $f$ induces a pro-isomorphism on these homotopy groups. Consequently, by Lemma 4.2 of [2, p. 79] $f$ induces an isomorphism

$$
H^{*}(X ; N) \cong \operatorname{colim} H^{*}\left(Y_{n} ; N\right)
$$

for any $\pi_{1} Y_{k}$-module $N$.

Now by a fundamental property of the plus construction [4, p. 313], for any $\pi_{1} Y_{k}^{+}$-module $M$ the inclusion $Y_{k} \rightarrow Y_{k}^{+}$induces an isomorphism

$$
H^{*}\left(Y_{k} ; M\right) \stackrel{\cong}{\rightleftarrows} H^{*}\left(Y_{k}^{+} ; M\right)
$$

so that we also obtain for any $\pi_{1} Y_{k}^{+}$-module $M$ an isomorphism

$$
H^{*}\left(X^{+} ; M\right) \cong \operatorname{colim} H^{*}\left(Y_{n}^{+} ; M\right)
$$

induced by $f^{+}$. As the towers $\left\{\pi_{1} X^{+}\right\}$and $\left\{\pi_{1} Y_{n}^{+}\right\}$are the towers of units in $\mathbf{Z}_{(p)}$ and in $\pi_{0} Q S_{(n)}$, respectively, $f^{+}$induces a pro-isomorphism between 
them, so we may apply Lemma 2.3 to complete the proof. In a nutshell, the proof is a process of pushing the weak pro-homotopy equivalence $f$ down to a weak pro-homotopy equivalence $f^{+}$.

Using informal language then, we say the tower $\{\widetilde{A}(*, p, n)\}$ converges to $A(*, p)$, the $K$-theory of the category of $p$-local finite spectra.

The following relative form of this result is perhaps easier to come to grips with and helps explain the sense in which the localization tower leads to a sort of fine structure on

$$
F=\operatorname{fibre}(A(*) \rightarrow K(\mathbf{Z})) .
$$

To simplify the notation put

$$
F(p, n)=\operatorname{fibre}\left(\tilde{A}(*, p, n) \rightarrow K\left(\mathbf{Z}_{(p)}\right)\right) .
$$

By the splitting result $[8$, p. 186] mentioned near the beginning of this note,

$$
F_{(p)}=\operatorname{fibre}\left(A(*, p) \rightarrow K\left(\mathbf{Z}_{(p)}\right)\right),
$$

where $F_{(p)}$ denotes the $p$-localization of $F$. Applying the five lemma in the category of pro-groups $[2$, p. 75$]$ one immediately obtains the following consequence of the theorem.

Corollary 2.9. With notation as above, the natural map

$$
\left\{F_{(p)}\right\} \rightarrow\{F(p, n)\}
$$

from the constant tower on $F_{(p)}$ is a weak pro-homotopy equivalence.

\section{REFERENCES}

1. A. K. Bousfield, The localization of spectra with respect to homology, Topology 18 (1979), 257-281.

2. A. K. Bousfield and D. M. Kan, Homotopy limits, completions, and localizations, Lecture Notes in Math., vol. 304, Springer-Verlag, New York, 1972.

3. M. J. Hopkins and D. C. Ravenel, A proof of the smash product conjecture (to appear).

4. J.-L. Loday, K-théorie algébrique et représentationes de groupes, Ann. Sci. École Norm. Sup. (4) 9 (1976), 309-377.

5. J. P. May, Simplicial objects in algebraic topology, Van Nostrand, Princeton, NJ, 1967.

6. D. C. Ravenel, Localization with respect to certain periodic homology theories, Amer. J. Math. 106 (1984), 351-414.

7. E. H. Spanier, Algebraic topology, McGraw-Hill, New York, 1966.

8. F. Waldhausen, Algebraic $K$-theory of spaces, localization, and the chromatic filtration of stable homotopy, Lecture Notes in Math., vol. 1051, Springer, New York, 1984, pp. 173196.

9. D. C. Ravenel, Life after the Telescope Conjecture (to appear). 0027

Department of Mathematics, University of Kentucky, Lexington, Kentucky 40506-

Current address: Department of Mathematics, Purdue University, West Lafayette, Indiana 47907-1395

E-mail address: mcclure@math.purdue.edu

Department of Mathematics, New Mexico State University, Las Cruces, New Mexico 88003

E-mail address: ross@nmsu.edu 